EUROPEAN ORGANIZATION FOR NUCLEAR RESEARCH

European Laboratory for Particle Physics

Large Hadron Collider Project

LHC Project Report 499

\title{
ADVANCED SUPERCONDUCTING TECHNOLOGY FOR GLOBAL SCIENCE: THE LARGE HADRON COLLIDER AT CERN
}

\author{
$\mathrm{Ph}$. Lebrun
}

\begin{abstract}
The Large Hadron Collider (LHC), presently in construction at CERN, the European Organisation for Nuclear Research near Geneva (Switzerland), will be, upon its completion in 2005 and for the next twenty years, the most advanced research instrument of the world's high-energy physics community, providing access to the energy frontier above $1 \mathrm{TeV}$ per elementary constituent. Re-using the 26.7-km circumference tunnel and infrastructure of the past LEP electron-positon collider, operated until 2000, the LHC will make use of advanced superconducting technology - high-field Nb-Ti superconducting magnets operated in superfluid helium and a cryogenic ultra-high vacuum system - to bring into collision intense beams of protons and ions at unprecedented values of center-of-mass energy and luminosity (14 TeV and $10^{34} \mathrm{~cm}^{-2} \cdot \mathrm{s}^{-1}$, respectively with protons). After some ten years of focussed $\mathrm{R} \& \mathrm{D}$, the LHC components are presently series-built in industry and procured through world-wide collaboration. After briefly recalling the physics goals, performance challenges and design choices of the machine, we describe its major technical systems, with particular emphasis on relevant advances in the key technologies of superconductivity and cryogenics, and report on its construction progress.
\end{abstract}

LHC Division

Invited paper at the CEC-ICMC 2001 Conference

16-20 July 2001, Madison, Wisconsin, USA

\footnotetext{
Administrative Secretariat

LHC Division

CERN

CH - 1211 Geneva 23

Switzerland

Geneva, 27 August 2001
} 


\title{
ADVANCED SUPERCONDUCTING TECHNOLOGY FOR GLOBAL SCIENCE: THE LARGE HADRON COLLIDER AT CERN
}

\author{
$\mathrm{Ph}$. Lebrun \\ CERN, European Organization for Nuclear Research, LHC Division \\ 1211 Geneva 23, Switzerland
}

\begin{abstract}
The Large Hadron Collider (LHC), presently in construction at CERN, the European Organisation for Nuclear Research near Geneva (Switzerland), will be, upon its completion in 2005 and for the next twenty years, the most advanced research instrument of the world's high-energy physics community, providing access to the energy frontier above $1 \mathrm{TeV}$ per elementary constituent. Re-using the 26.7-km circumference tunnel and infrastructure of the past LEP electron-positon collider, operated until 2000, the LHC will make use of advanced superconducting technology - high-field $\mathrm{Nb}$ - $\mathrm{Ti}$ superconducting magnets operated in superfluid helium and a cryogenic ultra-high vacuum system - to bring into collision intense beams of protons and ions at unprecedented values of center-ofmass energy and luminosity (14 TeV and $10^{34} \mathrm{~cm}^{-2} \cdot \mathrm{s}^{-1}$, respectively with protons). After some ten years of focussed R\&D, the LHC components are presently series-built in industry and procured through world-wide collaboration. After briefly recalling the physics goals, performance challenges and design choices of the machine, we describe its major technical systems, with particular emphasis on relevant advances in the key technologies of superconductivity and cryogenics, and report on its construction progress.
\end{abstract}

\section{INTRODUCTION}

The basic tools of elementary-particle physics, high-energy accelerators and large detectors, have developed in size, performance and complexity over the years to become major advanced engineering ventures, with budgets in the billion-dollar range and construction spreading over a decade, crystallising the efforts of thousands of physicists and engineers world wide, in industry, academia and a few regional centers such as CERN, the European Organisation for Nuclear Research near Geneva (Switzerland), funded by its twenty member states. Elementary-particle physics is the archetype of "big science" and the sheer size of its facilities, exceeding the funding capabilities of single nations as early 
as the 1960s, has pushed it into international collaboration, spurred both by necessity and by virtue of its goal as a quest for fundamental understanding of the universe around us. The most advanced project in this domain is the Large Hadron Collider (LHC) [1], a 26.7 $\mathrm{km}$ circumference accelerator based on high-field superconducting magnets operating in superfluid helium, presently under construction at CERN through a global collaboration involving all regions of the world active in the field [2]. Upon its completion in 2005, the LHC will bring into collision intense beams of protons and ions at unprecedented energy and luminosity (14 TeV center-of-mass and $10^{34} \mathrm{~cm}^{-2} \cdot \mathrm{s}^{-1}$, respectively for protons), thus opening a new window on the structure of matter and the basic forces of nature. The collisions will be produced and their products analysed in four large experiments located in underground caverns around the machine. FIGURE 1 shows the general layout of the LHC, while its main parameters as a proton collider are listed in TABLE 1. A view of the machine installed in the tunnel appears in FIGURE 2.

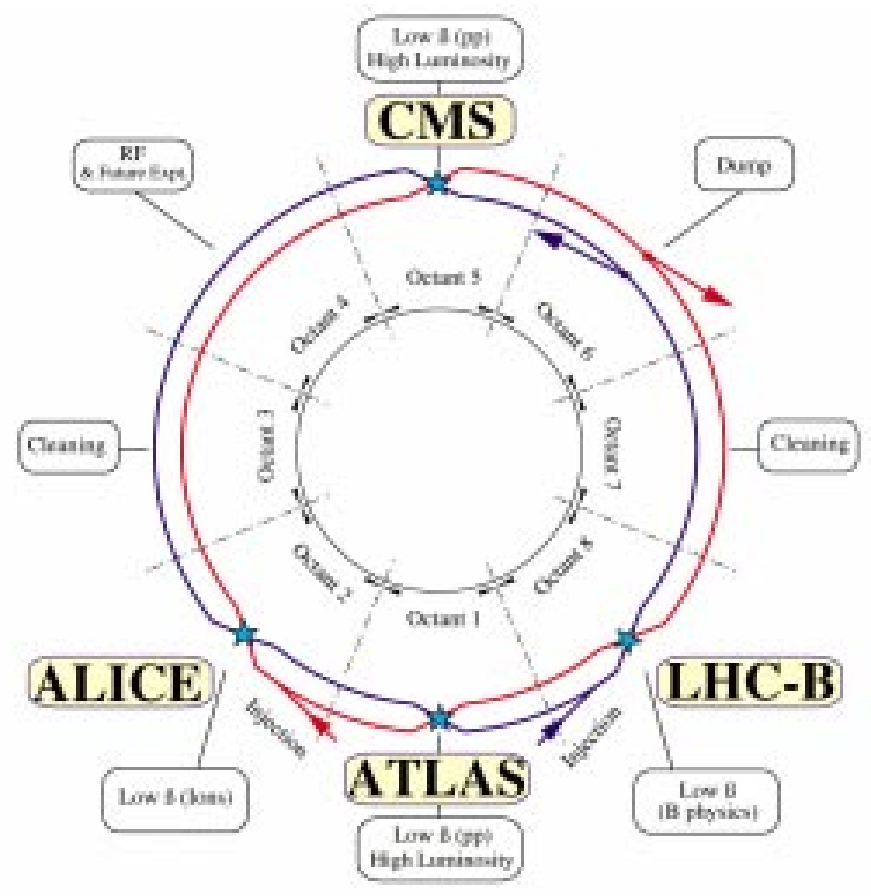

FIGURE 1. General layout of the LHC

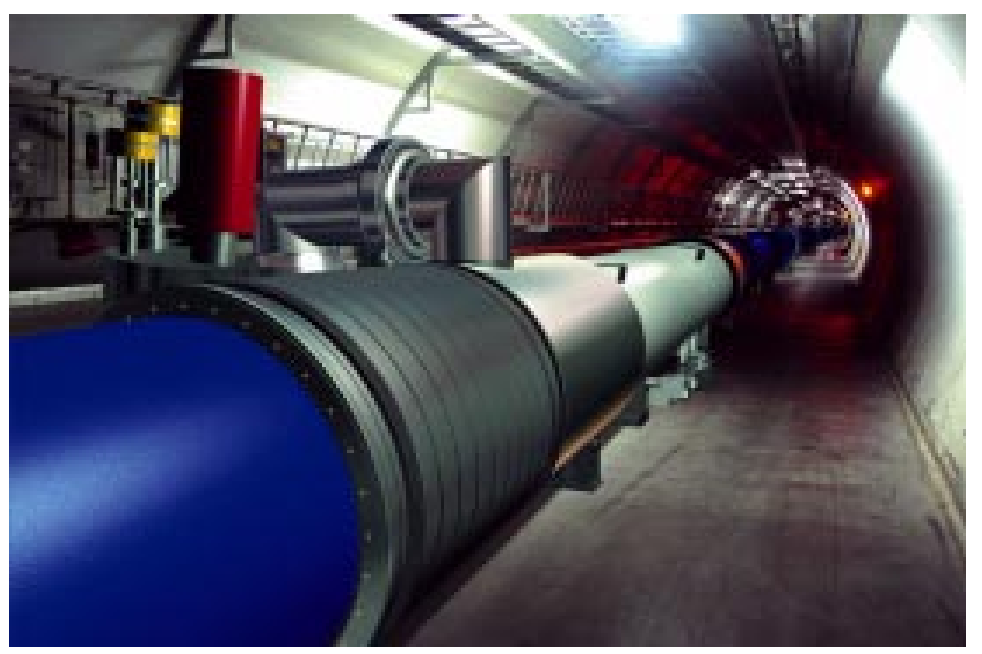

FIGURE 2. Artist's view of the LHC in its tunnel: the curvature is barely visible 
TABLE 1. Parameters of the LHC as proton collider

\begin{tabular}{lll}
\hline Energy at collision & 7 & $\mathrm{TeV}$ \\
Energy at injection & 450 & $\mathrm{GeV}$ \\
Dipole field at 7 TeV & 8.33 & $\mathrm{~T}$ \\
Coil inner diameter & 56 & $\mathrm{~mm}$ \\
Distance between aperture axes $(1.9 \mathrm{~K})$ & 194 & $\mathrm{~mm}^{-2}$ \\
Luminosity & 1034 & $\mathrm{~cm}^{-1}$ \\
Beam current & 0.56 & $\mathrm{~A}$ \\
Bunch spacing & 7.48 & $\mathrm{~m}$ \\
Bunch separation & 24.95 & $\mathrm{~ns}$ \\
Number of particles per bunch & $1.1 \times 10^{11}$ & \\
Normalized transverse emittance (r.m.s.) & 3.75 & $\mu \mathrm{m}$ \\
Total crossing angle & 300 & $\mu \mathrm{rad}$ \\
Luminosity lifetime & 10 & $\mathrm{~h}$ \\
Energy loss per turn & 6.7 & $\mathrm{keV}$ \\
Critical photon energy & 44.1 & $\mathrm{eV}$ \\
Total radiated power per beam & 3.8 & $\mathrm{~kW}$ \\
Stored energy per beam & 350 & $\mathrm{MJ}$ \\
\hline
\end{tabular}

\section{PHYSICS GOALS [3]}

Down to the de Broglie scale of $10^{-18} \mathrm{~m}$, corresponding to the $\mathrm{TeV}$ energy per elementary constituent of the LHC, nature around us is described by the so-called Standard Model: matter is built up of combinations of three families of fermions of different types (quarks, electrons, neutrinos) subject to several forces mediated by bosons (photons, "weak" bosons, gluons). In spite of its remarkable success as a descriptive and predictive theory (with a precision of $10^{-3}$ or better), the Standard Model still shows several shortcomings. The origin of the particle masses and their distribution, which spans more than twelve orders of magnitude, are neither predicted nor explained. A possible process to endow particles with masses is their coupling with a particular field permeating space, the Higgs field, which would be mediated by the Higgs boson. Theoretical considerations and experimental searches for the Higgs boson indicate that its mass range would fall between $115 \mathrm{GeV}$ and about $1 \mathrm{TeV}$, well within reach of the LHC.

However, the Standard Model including the Higgs mechanism may well not be the ultimate theory. The concept of Grand Unified Theories, which predict the unification of the strengths of electromagnetic, weak and strong interactions at very high energy, would require amending the Standard Model to include other particles, "supersymmetric" to the known ones. Although this unification would only occur at energies far above those of the LHC collisions, its consequences could appear as new physics down to the TeV range accessible to the machine. The LHC will also be able to produce at enormous rates, the known particles discovered with other accelerators, providing large statistics permitting precision measurements that will confirm the Standard Model or require its modification. Moreover, the LHC will explore through dedicated experiments the origin of matterantimatter assymmetry, as well as the deconfinement of the quarks and gluons in a "quarkgluon plasma", such as occurred in the early universe. Finally, by providing a resolution power an order of magnitude higher than previous accelerators, the LHC constitutes a true discovery machine, which may reveal totally unexpected physics. More than 4000 physicists from all over the world are coordinating their efforts to design, construct and operate four large detectors which will be installed around the collision points: ATLAS, CMS, ALICE and LHCb (FIGURE 1). 


\section{PERFORMANCE CHALLENGES [4]}

The history of particle accelerators since the 1930s is a race towards higher energy, illustrated on the Livingston diagram [5], which shows a sustained exponential growth over many orders of magnitude, from the tin-can size of E.O. Lawrence's first cyclotron to the footprint of present projects, comparable to that of a regional capital. Although the growth in size appears the most spectacular, the development in performance is even faster, thanks to timely implementation of emerging technologies, each superseding the concurrent established solution as it tends to show saturation, and thus allowing to contain the corresponding increase in investment cost. The most recent part of the diagram (FIGURE 3) illustrates the emergence and widespread diffusion of superconducting technology in high-energy accelerators over the last twenty years. For a given beam energy, the size of circular hadron accelerators is in inverse proportion to the field in the bending magnets. The first superconducting machines to be built operate around $5 \mathrm{~T}$, a significant gain with respect to the $2 \mathrm{~T}$ limit of conventional iron-cored magnets. To get the most out of reusing the existing $26.7 \mathrm{~km}$ circumference tunnel which previously housed the LEP collider at CERN, the LHC set the goal of operating in the 8 to $10 \mathrm{~T}$ range, permitting to reach a beam energy of $7 \mathrm{TeV}$. Moreover, the reuse of existing CERN accelerators as preinjectors imposes an injection energy of $450 \mathrm{GeV}$, thus requiring a large dynamic range in energy.

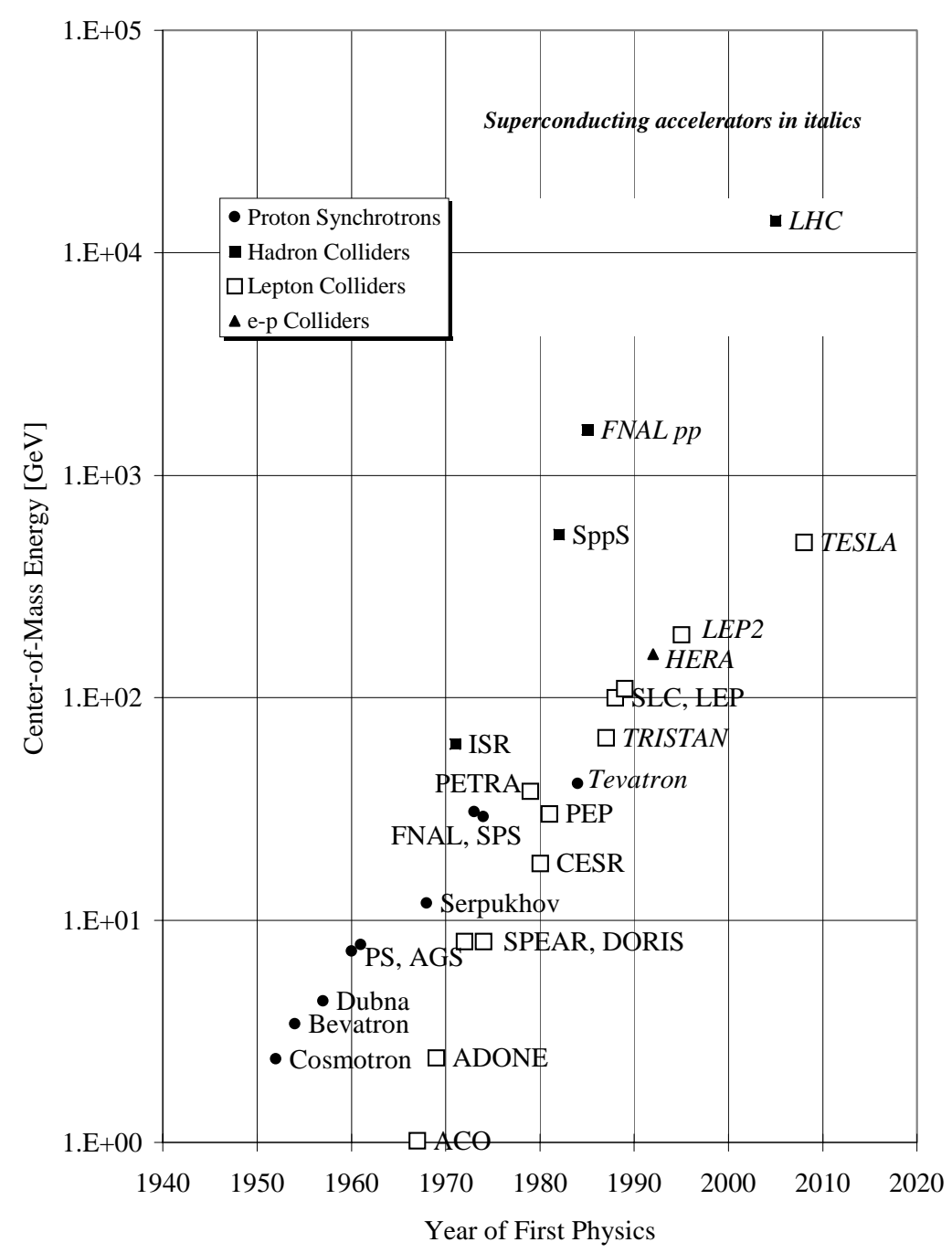

FIGURE 3. Superconductivity and the energy frontier in accelerators 
An equally demanding requirement is the high luminosity of the machine, essential to provide sufficient statistics for rare physics events. This challenge, which represents almost a hundred-fold increase with respect to the present state-of-the-art, is met by accelerating and colliding high-intensity, low-emittance beams, composed of several thousand bunches with fast repetition rate. Losing only a p.p.m. of the circulating beams in the superconducting magnets would deposit sufficient energy to quench the windings: the LHC would not work without a very efficient beam loss monitoring and collimation system. Other consequences of the high-intensity beams are the power deposition by synchrotron radiation and dissipation of beam image currents, intercepted at higher temperature by beam screens before they reach the $1.9 \mathrm{~K}$ level, as well as the risk of resonant acceleration of electrons emitted from the vacuum chamber wall ("electron cloud"), leading to beam instability and additional power deposition [6]. Finally, the fact that most of the collision products are emitted at low angle with respect to the circulating beams induces high radiation and heat loads in the experimental areas and insertions.

\section{HIGH-FIELD SUPERCONDUCTING MAGNETS}

The main technological challenge of the LHC is the development and industrial production of 1232 superconducting main dipoles [7] operating at $8.3 \mathrm{~T}, 400$ superconducting main quadrupoles [8] producing gradients of $223 \mathrm{~T} \mathrm{~m}^{-1}$, and several thousand other superconducting magnets [9], for correcting multipole errors, steering and colliding [10] the beams, and increasing luminosity in collision [11,12]. All these magnets (TABLE 2), which must produce a controlled field with a precision of $10^{-4}$, are presently being series-produced by industry in Europe, India, Japan and the USA.

TABLE 2. Superconducting magnets in the LHC

\begin{tabular}{lll}
\hline Type & Quantity & Purpose \\
\hline MB & 1232 & Main dipole \\
MQ & 400 & Main quadrupole \\
MSCB & 376 & Combined chromaticity and closed-orbit corrector \\
MCS & 2464 & Sextupole for correcting dipole persistent currents \\
MCDO & 1232 & Octupole/decapole for correcting dipole persistent currents \\
MO & 336 & Landau octupole for instability control \\
MQT & 256 & Trim quadrupole for lattice correction \\
MCB & 266 & Orbit correction dipole \\
MQM & 100 & Dispersion suppressor quadrupole \\
MQX & 32 & Low- $\beta$ insertion quadrupole \\
MQY & 20 & Enlarged-aperture quadrupole \\
\hline
\end{tabular}

A specific feature of the main dipoles, a cross-section of which appears in FIGURE 4, is their twin-aperture design. To produce the anti-parallel fields required for bending the counter-rotating beams along their paths in the tunnel, the collider needs two separate magnetic channels. This is conventionally achieved by installing side by side two separate strings of magnets, each in their own cryostat. In the LHC, two sets of windings are combined in a common mechanical and magnetic structure to constitute twin-aperture magnets, a more compact and efficient solution, as the return flux of one aperture contributes to increasing the field in the other. The field level and quality in the magnet apertures are produced by winding Rutherford-type multi-strand keystoned cables, in a graded two-layer $\cos \theta$ geometry. The very large electromagnetic forces acting on the conductors are reacted 


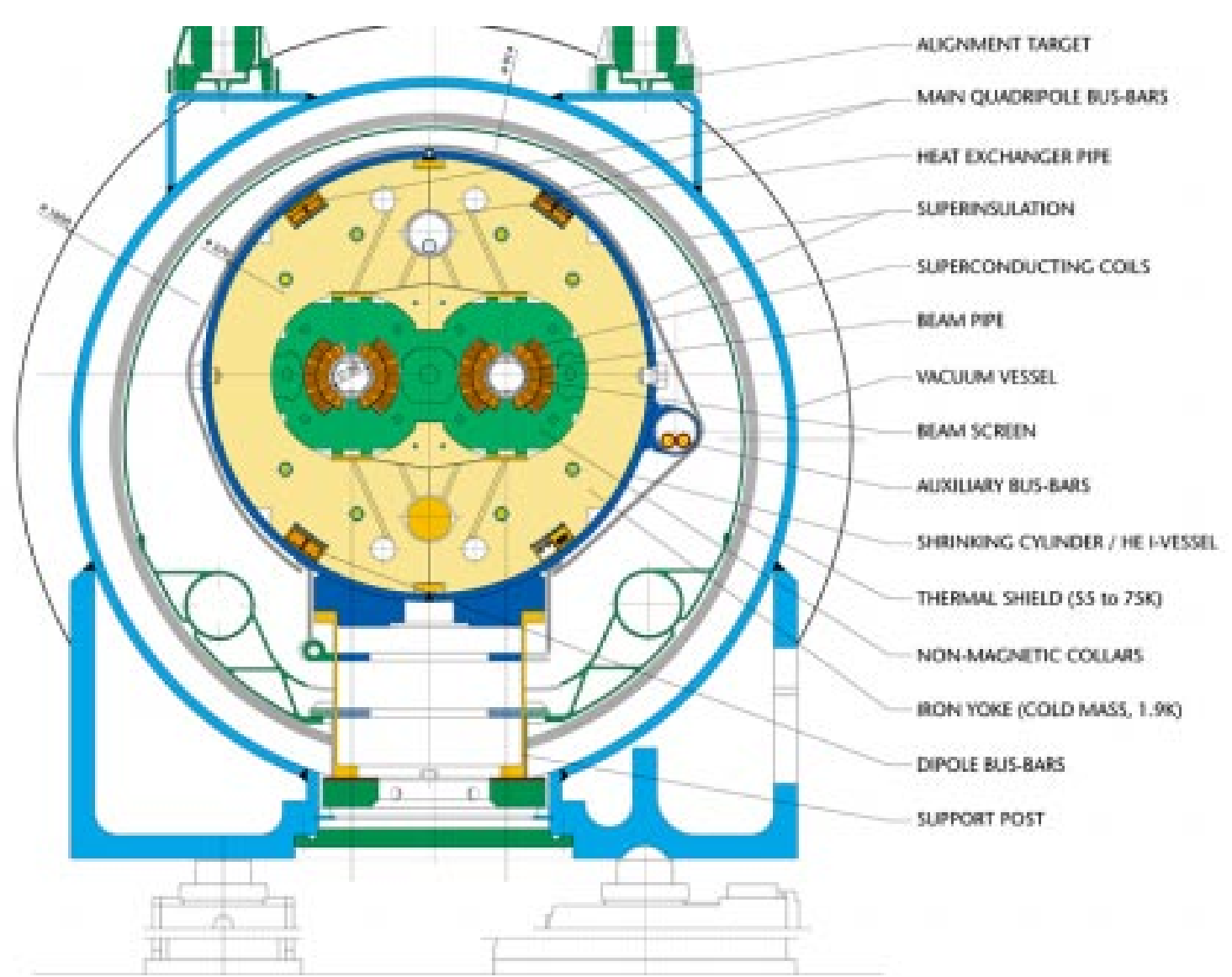

FIGURE 4. Transverse cross section of twin-aperture dipole in its cryostat

by non-magnetic collars resting against the stiff iron yoke, contained in an all-welded shrinking cylinder which also acts as helium enclosure and pressure vessel.

The decreasing critical current density of $\mathrm{Nb}$ - $\mathrm{Ti}$ alloys with increasing induction (FIGURE 5) precludes the use of this material for building high-field magnets operated in normal helium at $4.5 \mathrm{~K}$. The alternative $\mathrm{A} 15$ compounds, such as $\mathrm{Nb}_{3} \mathrm{Sn}$, are however plagued by their difficult implementation (wind-and-react process), limited industrial availability - the LHC requires some 1250 tonnes of superconductor - and high cost. CERN therefore decided to base the LHC project on the use of $\mathrm{Nb}$-Ti operating in superfluid helium at $1.9 \mathrm{~K}$ where it retains sufficient current-carrying capacity for building magnets up to about $10 \mathrm{~T}$. This technique, pioneered in the 1980s in the Tore Supra tokamak and other high-field magnets [13], is applied for the first time to the magnets of a large accelerator. The LHC magnets must preserve their field quality over a large dynamic range, in particular at low level when persistent currents in the superconductor produce remanence. This requires the diameter of the $\mathrm{Nb}$ - $\mathrm{Ti}$ filaments in the cable strands not to exceed $7 \mu \mathrm{m}$, a technical/economical compromise which can be obtained by single-stack billet manufacturing.

Following a decade of development and model work, final prototypes magnets built in industry have permitted the validation of technical design choices and manufacturing techniques, thus leading the way for the adjudication of pre-series and series contracts for the dipoles, quadrupoles and correctors, the production of which has started and is expected to spread over the next four years (FIGURE 6). 


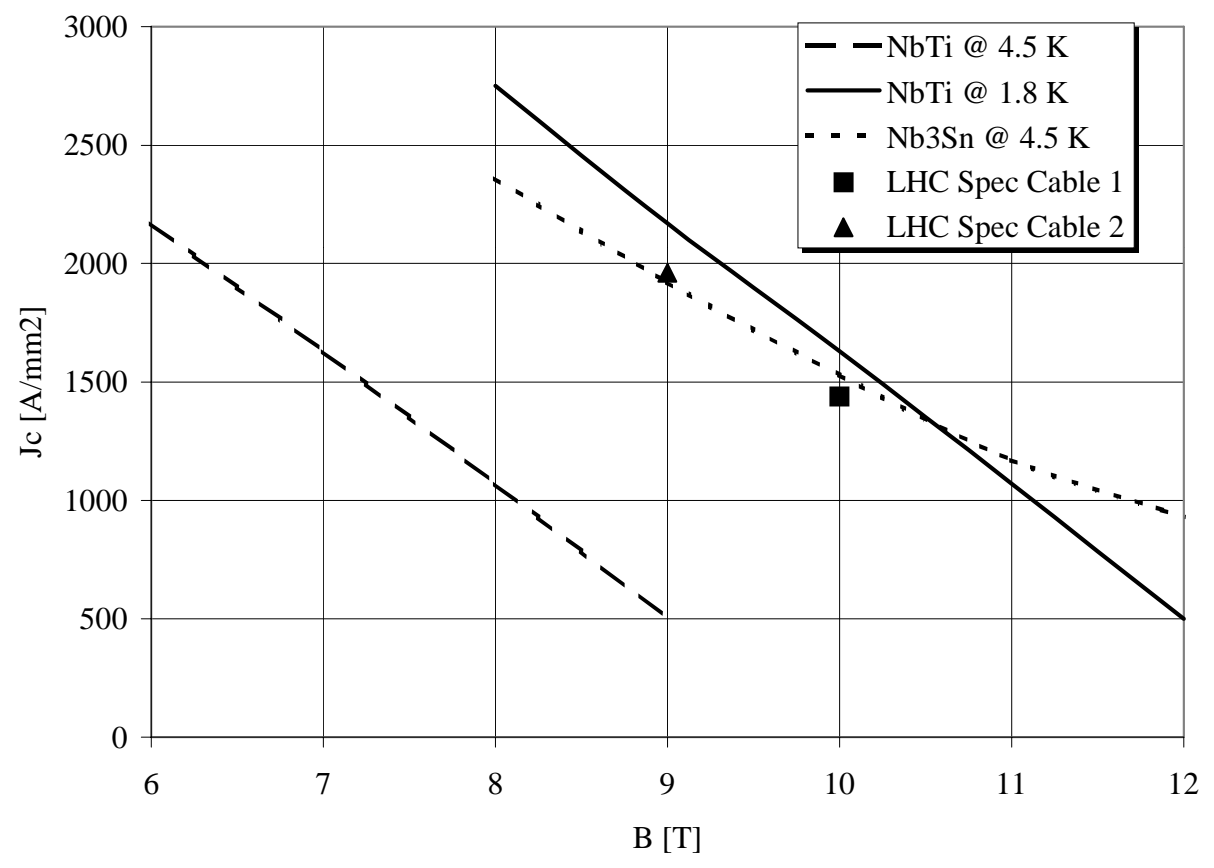

FIGURE 5. Critical current density of technical superconductors

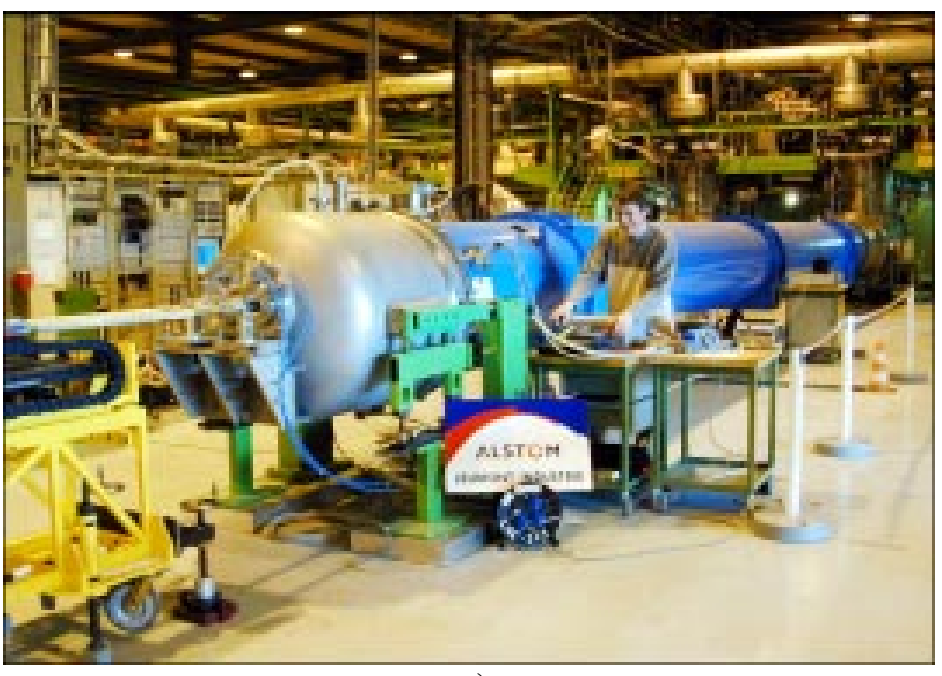

a)

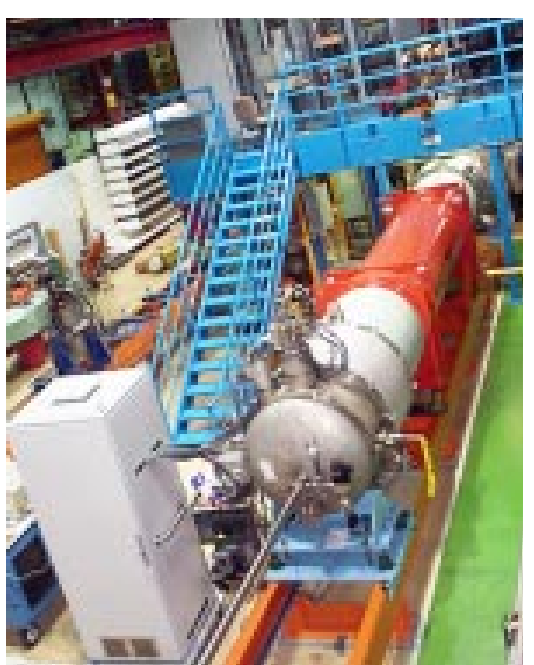

b)

FIGURE 6. First pre-series superconducting magnets under test. a) Main dipole at CERN; b) Low- $\beta$ quadrupole at Fermilab

\section{HIGH-TEMPERATURE SUPERCONDUCTOR CURRENT LEADS}

Powering the magnet circuits in the LHC will require feeding $3.4 \mathrm{MA}$ into the cryogenic environment, some $80 \%$ of which is in the form of currents of several kA, culminating with the $13 \mathrm{kA}$ rating of the main dipole and quadrupole circuits. Using resistive vapour-cooled current leads for this purpose would result in a heavy liquefaction load, exergetically equivalent to the production of an additional LHC sector refrigerator. The advent of quasi-industrial HTS materials, combined with the favourable cooling conditions provided by the availability of $20 \mathrm{~K}$ gaseous helium in the LHC cryogenic 
system, renders the use of HTS-based current leads very attractive. With a comfortable temperature difference to extract the heat from the resistive section in a compact heat exchanger, this allows operation of the upper end of the HTS section below $50 \mathrm{~K}$, a temperature at which the presently available materials, e.g., BSCCO 2223 in a silver matrix, exhibit much higher critical current density than at the usual $80 \mathrm{~K}$ provided by liquid nitrogen cooling. The thermodynamic rationale for using such HTS-based current leads is presented in TABLE 3, in comparison with conventional resistive vapour-cooled leads. While the heat reaching the lower end of the lead in liquid helium is reduced by an order of magnitude, the total exergy consumption, taking into account the load on the $20 \mathrm{~K}$ gaseous helium flow, is cut by a factor of about 3 .

TABLE 3. Performance of HTS-based current leads for the LHC, compared to resistive vapour-cooled leads

\begin{tabular}{llll}
\hline Lead type & & $\begin{array}{l}\text { Resistive, vapour-cooled } \\
(4 \text { to 300 K) }\end{array}$ & $\begin{array}{l}\text { HTS (4 to 50 K) } \\
\text { Resistive, gas cooled (50 to 300 K) }\end{array}$ \\
\hline Heat into LHe & {$[\mathrm{W} / \mathrm{kA}]$} & 1.1 & 0.1 \\
Total exergy consumption & {$[\mathrm{W} / \mathrm{kA}]$} & 430 & 150 \\
Electrical power from grid & {$[\mathrm{W} / \mathrm{kA}]$} & 1430 & 500 \\
\hline
\end{tabular}

After conducting tests on material samples, CERN has procured from industry and intensively tested prototypes of HTS-based current leads for $13 \mathrm{kA}$ and $0.6 \mathrm{kA}$, thus enabling us to demonstrate feasibility and performance of this solution, identify potential construction problems, address transient behaviour and control issues, and prepare the way for procurement of series units [14]. FIGURE 7 shows the electrical feedbox equipped with prototype $13 \mathrm{kA}$ and $0.6 \mathrm{kA}$ leads which feeds the LHC Test String [15].

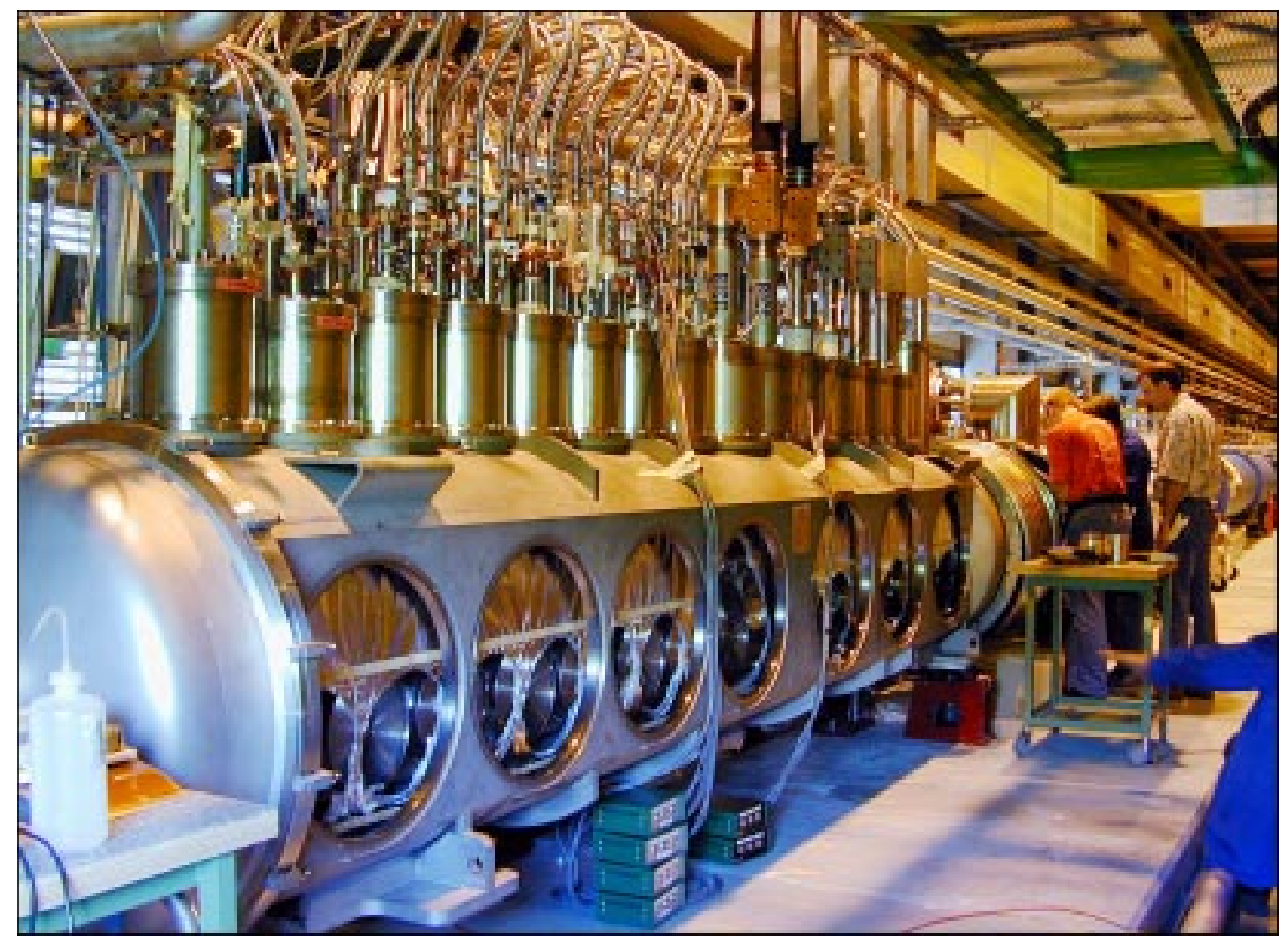

FIGURE 7. Electrical feedbox equipped with high-temperature superconductor current leads 


\section{SUPERFLUID HELIUM CRYOGENICS}

The prime reason for superfluid helium cooling of the LHC magnets is the lower operating temperature, and hence the increased working range of the superconductor. However, as the specific heat of the $\mathrm{Nb}$ - Ti alloy - and that of its copper stabilising matrix rapidly fall with decreasing temperature, the full benefit of the lower-temperature operation may only be reaped, in terms of stability margin, by making effective use of the particular transport properties of superfluid helium, both for extracting heat - whether steady or transient dissipation - from the magnet windings, and for transporting it over the long distances encountered in a large accelerator to the nearest heat sink [16]. The low bulk viscosity of superfluid helium enables it to permeate the magnet windings and make use of its very large specific heat - typically 2000 times that of the cable per unit volume - for buffering thermal disturbances, as well as of its huge thermal conductivity at moderate heat flux - 1000 times that of OFHC copper, peaking at $1.9 \mathrm{~K}$ - to transport it away. This requires the electrical insulation of the superconducting cable to preserve sufficient porosity and percolation paths while still fulfilling its demanding dielectric and mechanical functions. It has been obtained with a moderate filling factor of the keystoned cable and staggered wrappings of polyimide tape.

The large, but finite thermal conductivity of superfluid helium, which was used in earlier projects to transport the heat over distances of up to a few tens of meters, is insufficient given the heat loads and geometric configuration of the LHC, where every $3.3-\mathrm{km}$ long sector must be cooled from its dedicated refrigerator. Furthermore, the thermodynamic penalty of low-temperature operation limits the overall temperature drop for heat extraction and transport to a mere $0.1 \mathrm{~K}$. The LHC magnets operate in static baths of pressurised superfluid helium, a single-phase, quasi-isothermal medium, cooled by continuous heat exchange with flowing saturated superfluid helium, the latent heat of vapourisation of which provides a quasi-isothermal heat sink (FIGURE 8). This cooling scheme, which involves two-phase flow of superfluid helium in near-horizontal tubes, has been intensively studied on test loops and validated on a full-scale prototype magnet string [17]. It is implemented in cryogenic loops extending over $107 \mathrm{~m}$, the length of a lattice cell; all loops are fed in parallel from each cryogenic plant over the $3.3 \mathrm{~km}$ sector length through a compound cryogenic distribution line [18] running along the cryomagnets in the tunnel.

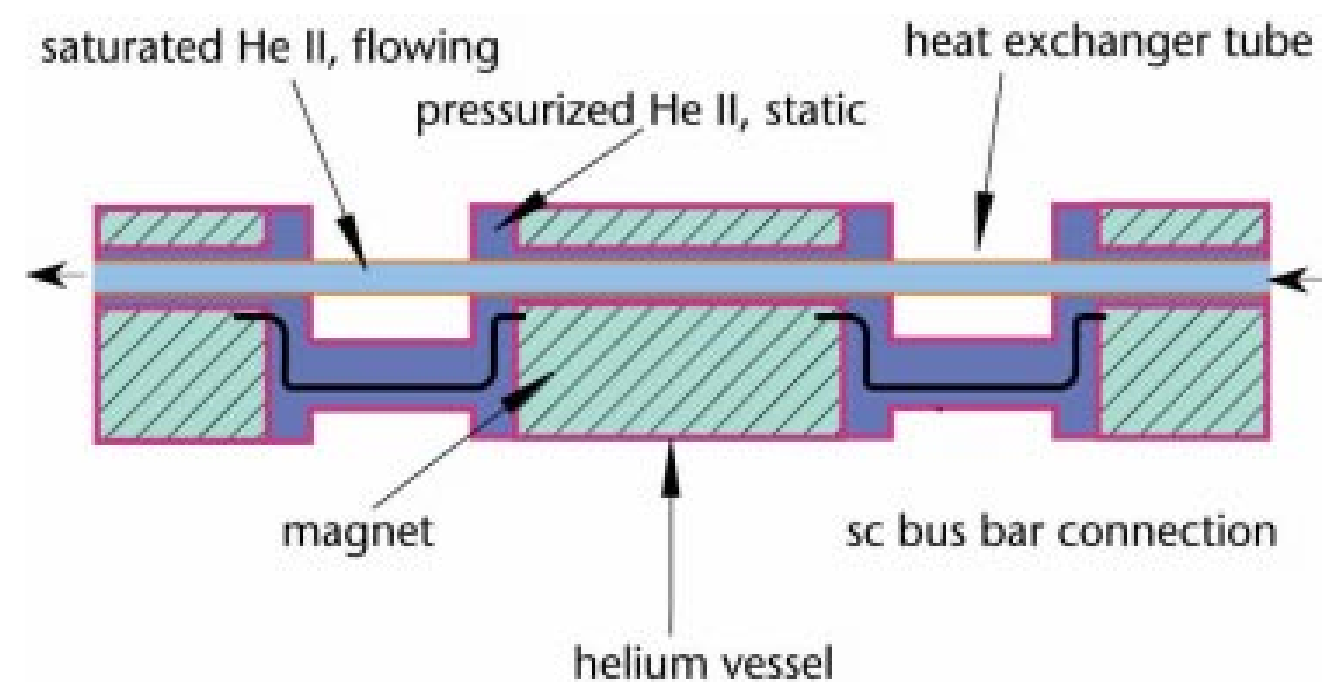

FIGURE 8. Principle of the LHC magnet cooling scheme 
The high thermodynamic cost of refrigeration at low temperature requires careful management of the system heat loads. In particular, it is essential to try and intercept heat at the highest possible temperature, so that only residuals reach the costly $1.9 \mathrm{~K}$ level. This has been achieved by the combined use of intermediate shielding, multilayer insulation and conduction intercepts in the design of the cryostats (FIGURE 4), as well as by the installation of beam screens, cooled between 5 and $20 \mathrm{~K}$ by supercritical helium, for absorbing the largest fraction of the beam-induced heat loads. To cope with its heat loads, the LHC will require eight large helium cryogenic plants, each producing a mixed duty of liquefaction and refrigeration at different temperatures, with an equivalent capacity of 18 $\mathrm{kW} @ 4.5 \mathrm{~K}$ and a coefficient of performance (c.o.p.) of $230 \mathrm{~W} / \mathrm{W}$ [19]. The coldbox of the first LHC cryogenic plant, presently undergoing reception tests at CERN, is shown in FIGURE 9.

In view of the low saturation pressure of helium at $1.8 \mathrm{~K}$, the compression of high flow-rates of helium vapour over a pressure ratio of 80 , can only be practically achieved by means of multi-stage cold hydrodynamic compressors (FIGURE 10). This technology, together with that of low-pressure heat exchangers, had to be developed specifically for this purpose, and integrated into novel thermodynamic cycles making the best possible use of the available components in order to minimise irreversibilities [20]. Following development and prototyping of the critical components, and detailed thermodynamic studies conducted in partnership with industry, eight $2400 \mathrm{~W} @ 1.8 \mathrm{~K}$ refrigeration units have been ordered from two companies, and the first one delivered to CERN for reception tests. The overall c.o.p. of these units, once connected to the conventional $4.5 \mathrm{~K}$ helium refrigerators, is expected to be around $900 \mathrm{~W} / \mathrm{W}$.

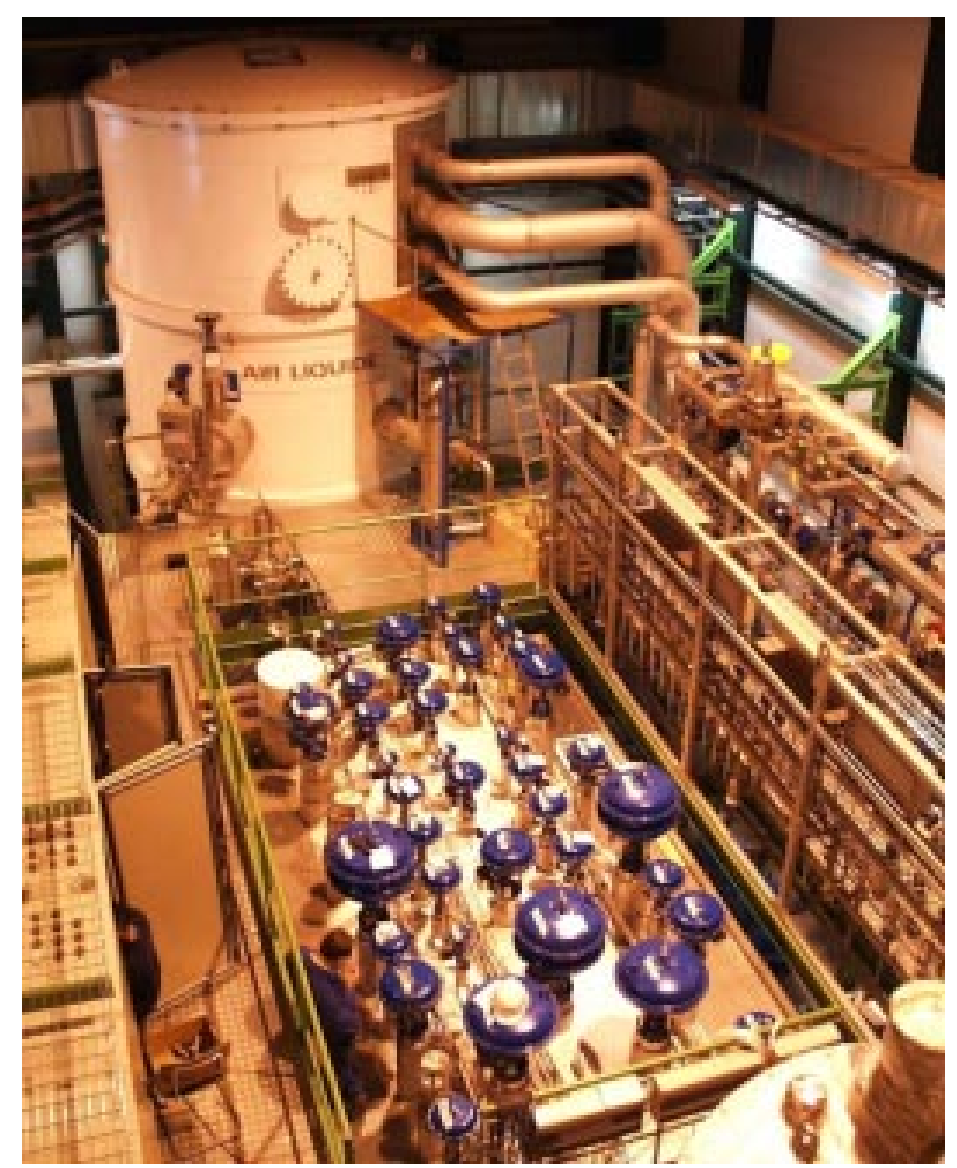

FIGURE 9. Coldbox of first $18 \mathrm{~kW} @ 4.5 \mathrm{~K}$ helium refrigerator 


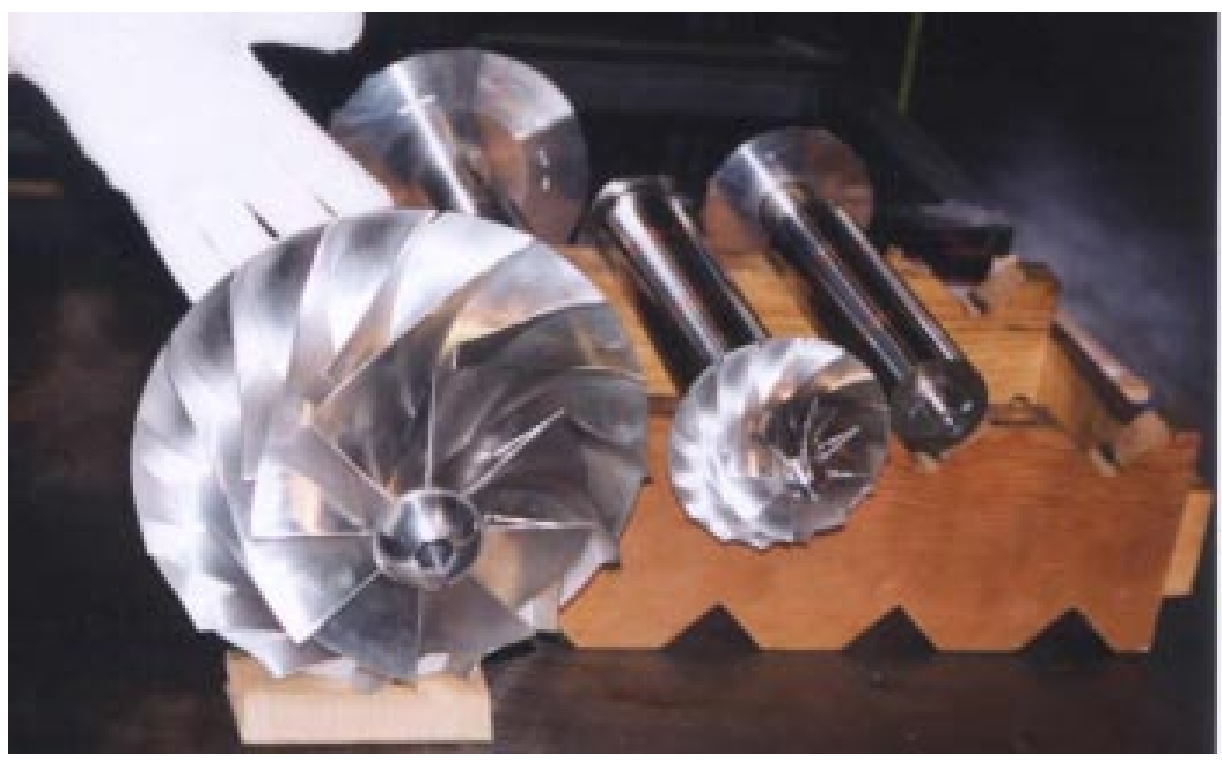

FIGURE 10. Impellers of cold compressors for the first $2.4 \mathrm{~kW} @ 1.8 \mathrm{~K}$ refrigeration unit

\section{CONCLUSION}

After a decade of focussed R\&D, the LHC construction is now in full swing [21]. Industrial contracts have been awarded and are under execution for the procurement of some 7000 superconducting magnets and of the largest helium cryogenic system ever built. Although located at CERN and basically funded by its twenty member states, the project, which will serve the world's high-energy physics community, is built through global collaboration, with special contributions from Canada, India, Japan, Russia and the USA. A full-scale test of the first sector is due by 2004, and colliding beams for physics are expected to be available from 2006 onwards.

\section{ACKNOWLEDGEMENTS}

The work reported here is mainly that of the LHC division at CERN. Fruitful collaboration with our colleagues in the laboratories of the contributing countries is gratefully acknowledged. Construction of the numerous high-technology components and systems is only possible thanks to the competence and sustained interest of specialised industry.

\section{REFERENCES}

1. The LHC Study Group, The Large Hadron Collider, Conceptual Design, CERN/AC/95-05, 1995.

2. Lebrun, Ph., "The Large Hadron Collider, a megascience project", in Superconducting Materials for High-energy Colliders, Proc. 38th INFN Eloisatron Workshop, edited by L. Cifarelli \& L. Maritato, World Scientific, 2001, pp. 11-20.

3. Gianotti, F., "Collider physics: LHC", in Proc. 1999 European School of High-Energy Physics, edited by A. Olchevski, CERN 2000-007, 2000, pp. 219-244.

4. Evans, L.R., "LHC Accelerator Physics and Technology Challenges", in Proc. PAC99, edited by A. Luccio \& W.W. MacKay, IEEE Piscataway, New Jersey, USA, 1999, pp. 21-25.

5. Livingston, M.S., High-energy accelerators, Interscience Publishers Inc., New-York, USA, 1954. 
6. Gröbner, O., "The LHC Vacuum System", in Proc. PAC97, edited by M. Comyn, M.K. Craddock \& M. Reiser, IEEE Piscataway, New Jersey, USA, 1998, pp. 3542-3546.

7. Wyss, C., "The LHC Magnet Programme: from Accelerator Physics Requirements to Production in Industry", in Proc. EPAC2000, edited by J.L. Laclare et al., Austrian Academy of Science Press, Vienna, Austria, 2000, pp. 207-211.

8. Billan, J. et al., "Performance of the Prototypes and Start-up of Series Fabrication of the LHC Arc Quadrupoles", paper presented at PAC2001, Chicago, USA, 2001.

9. Siegel, N., "Overview of LHC Magnets other than the Main Dipoles", in Proc. EPAC2000, edited by J.L. Laclare et al., Austrian Academy of Science Press, Vienna, Austria, 2000, pp. 23-27.

10. Willen, E. et al., "Superconducting Dipole Magnets for the LHC Insertion Regions", in Proc. EPAC2000, edited by J.C. Laclare et al., Austrian Academy of Science Press, Vienna, Austria, 2000, pp. 2187-2189.

11. Shintomi, T. et al., "Progress of LHC Low- $\beta$ Quadrupole Magnets at KEK", IEEE Trans. Appl. Superconductivity 11, pp. 1562-1565 (2001).

12. Andreev, N. et al., "Status of the LHC Inner Triplet Quadrupole Program at Fermilab", IEEE Trans. Appl. Superconductivity 11, pp. 1558-1561 (2001).

13. Claudet, G. \& Aymar, R., "Tore Supra and He-II Cooling of Large High-field Magnets", in Adv. Cryo. Eng. 35A, edited by R.W. Fast, Plenum, New York, 1990, pp. 55-67.

14. Ballarino, A., "High-temperature Superconducting Current Leads for the Large Hadron Collider", IEEE Trans. Appl. Superconductivity 9, pp. 523-526 (1999).

15. Bordry, F. et al., "The Commissioning of the LHC Test String 2", paper presented at PAC2001 Chicago, USA, 2001.

16. Lebrun, Ph., "Cryogenics for the Large Hadron Collider", IEEE Trans. Appl. Superconductivity 10, pp. 1500-1506 (2000).

17. Lebrun, Ph. et al., "Cooling Strings of Superconducting Devices below 2 K: the Helium II Bayonet Heat Exchanger", in Adv. Cryo. Eng. 43A, edited by P. Kittel, Plenum, New York, 1998, pp. 419-426.

18. Erdt, W. et al., "The LHC Cryogenic Distribution Line: Functional Specification and Conceptual Design", in Adv. Cryo. Eng. 45B, edited by Q.-S. Shu, Kluwer Academic/Plenum, New York, 2000, pp. 1387-1394.

19. Claudet, S. et al., "Economics of Large Helium Cryogenic Systems: Experience from Recent Projects at CERN", in Adv. Cryo. Eng. 45B, edited by Q.-S. Shu, Kluwer Academic/Plenum, New York, 2000, pp. 1301-1308.

20. Tavian, L., "Large Cryogenic Systems at 1.8 K", in Proc. EPAC2000, edited by J.L. Laclare et al., Austrian Academy of Science Press, Vienna, Austria, 2000, pp. 212-216.

21. Ostojic, R., "Status and Challenges of LHC Construction", invited paper at PAC2001, Chicago, USA, 2001. 